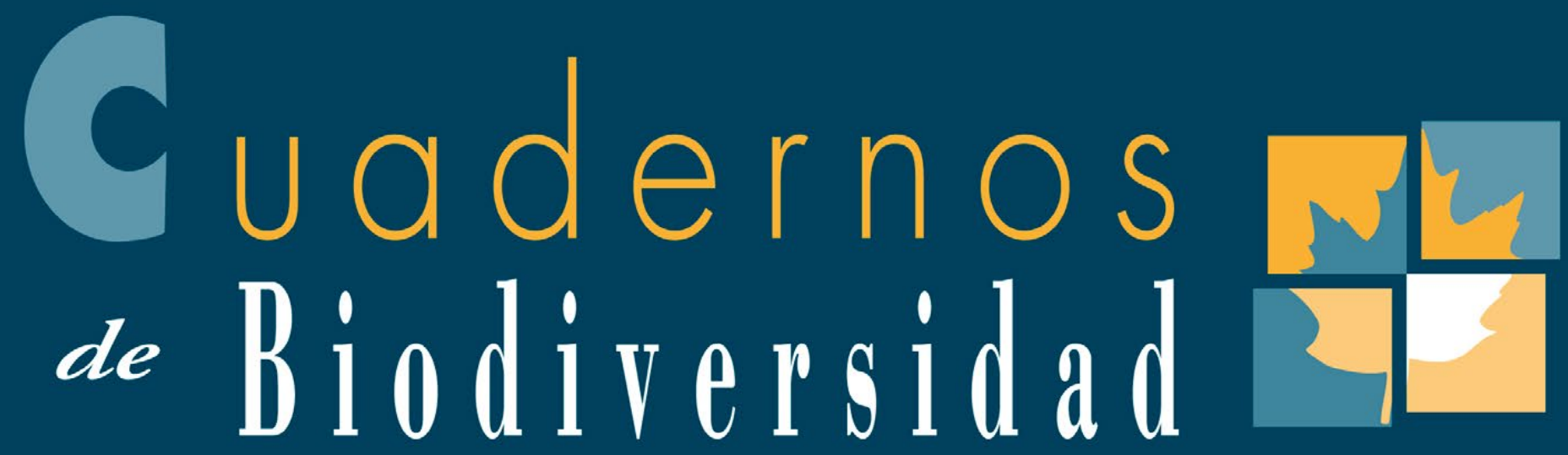

Cuadernos de biodiversidad número $47 \cdot 2015$
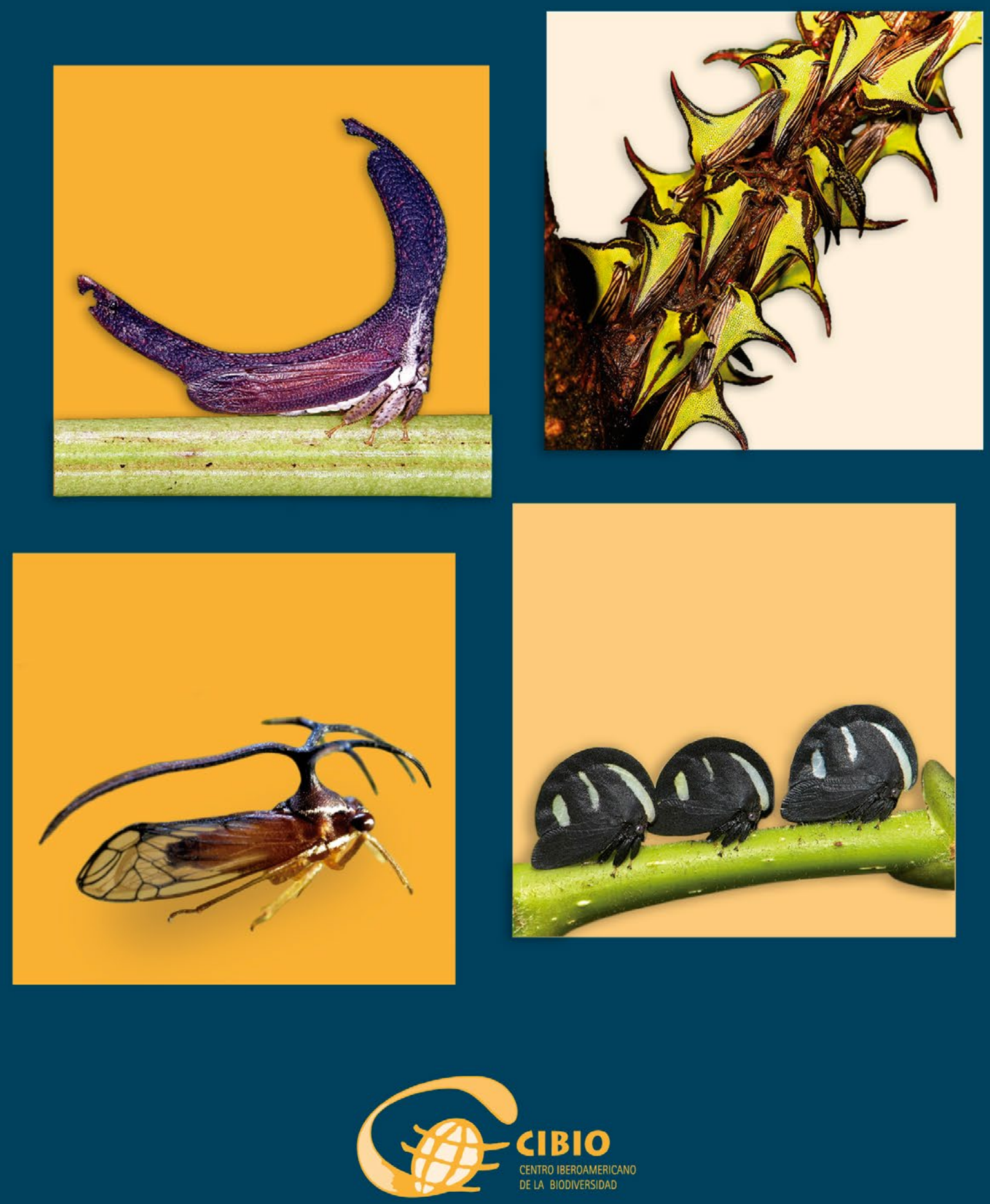

Universitat d'Alacant
Universidad de Alicante 


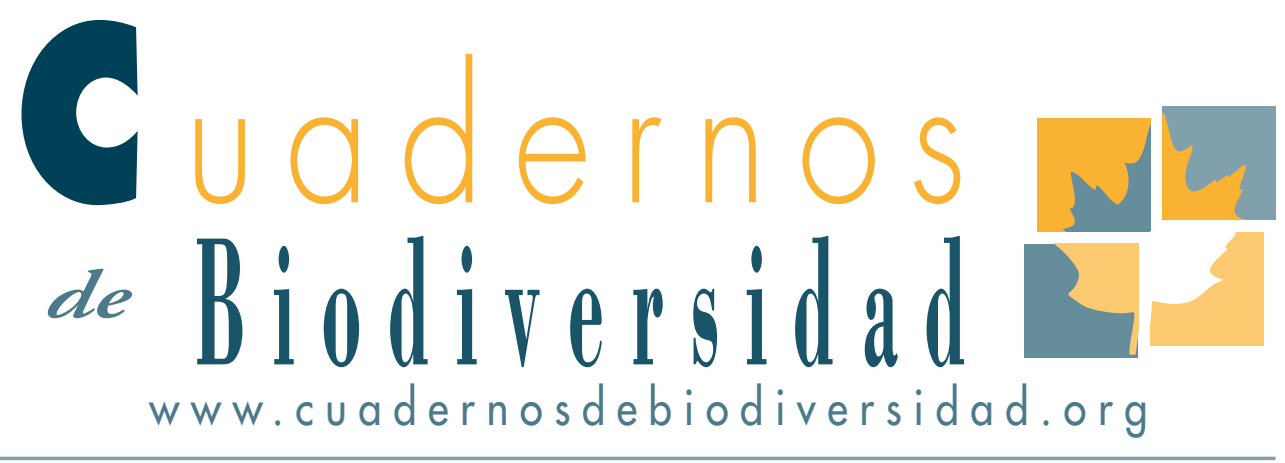

\section{El Museo de la Biodiversidad celebra su décimo aniversario}

\section{A. Eslava y M. Vila}

MUSEO DE LA BIODIVERSIDAD. AYUNTAMIENTO DE IBI. ALICANTE

EMAIL: MEDIOAMBIENTE@IBI.ES, MUSEO.BIO@IBI.ES

El Museo de la Biodiversidad creado hace ya una década por el Centro Iberoamericano de la Biodiversidad (CIBIO) de la Universidad de Alicante, tiene como objetivo principal el mostrar a la sociedad la diversidad de organismos y ecosistemas, buscando poner de manifiesto cuales son las principales amenazas que sufren a nivel mundial. Por todo ello, desde el museo se impulsa la difusión de los valores de la conservación de la biodiversidad convirtiéndose en un espacio de reflexión, donde se transmiten ideas e iniciativas para su conservación. A través de sus diferentes salas se desarrolla una muestra expositiva constituida por ejemplares procedentes de donaciones de otros museos públicos (MNCNM) y de particulares, proyectos científicos y material requisado por las autoridades administrativas responsables de la vigilancia del tráfico ilegal de especies.

Desde que surgió la idea de crear este espacio expositivo, el Excmo. Ayuntamiento de Ibi (Alicante), apostó decididamente por apoyar esta iniciativa, facilitando y posibilitando su instalación en dicha localidad, compromiso que con el tiempo se ha consolidado.
El Museo de la Biodiversidad abrió sus puertas al público por primera vez el 30 de septiembre de 2004, y sin dejar de crecer desde entonces, ha celebrado su décimo aniversario el pasado el 25 de octubre de 2014, con la participación activa de cientos de visitantes que pudieron vivir una jornada festiva jalonada por numerosas actividades.

El 7 de octubre se presentó a la prensa la nueva fase de ampliación del museo con la apertura de la nueva sala sobre "La Sabana Africana" y una nueva exposición temporal que bajo el título "Los Misterios de la Sabana" se podrá visitar hasta mediados de enero de 2015 (Foto 1). La nueva sala acoge 50 piezas naturalizadas de gran valor histórico depositadas por el Museo Nacional de Ciencias Naturales de Madrid (CSIC) gracias al convenio de colaboración firmado por dicho Museo, la Universidad de Alicante y el Ayuntamiento de Ibi para el desarrollo conjunto de actividades científicas, expositivas y divulgativas. Por otra parte, la exposición temporal ha sido posible gracias a la colaboración de diversos particulares que han cedido temporalmente animales naturalizados y objetos que forman parte de la cultura de las etnias que habitan en la sabana. 
Han sido muchas las actividades de celebración del X Aniversario a lo largo del mes de octubre, pero una de las más destacables quizás ha sido la denominada Una Noche en el Museo que convocó en las salas del Museo a 60 niños de corta edad que cargados con sus sacos de dormir y mochilas llenas de mucha ilusión, pasaron la noche entre vitrinas, objetos y naturaleza recreada (Foto 1 ). Se realizó una divertida visita por las colecciones, en la que todos los participantes se disfrazaron de los animales más representativos del museo. Luego, en la nueva sala de "La Sabana Africana", se introdujeron en los misterios de la sabana, sus características, biodiversidad y recursos que ofrece este ecosistema a los grupos humanos que la habitan (Foto 2). En esta sala se muestran las principales amenazas que afectan a la biodiversidad de la sabana y a los grupos étnicos que la habitan. Los participantes completaron la actividad con un taller sobre los misterios de África, lo que permitió a los niños convertirse en jefes de las tribus Mursi, Himba o Bosquimanos para terminar durmiéndose rodeados de animales después de una divertida Ginkana.

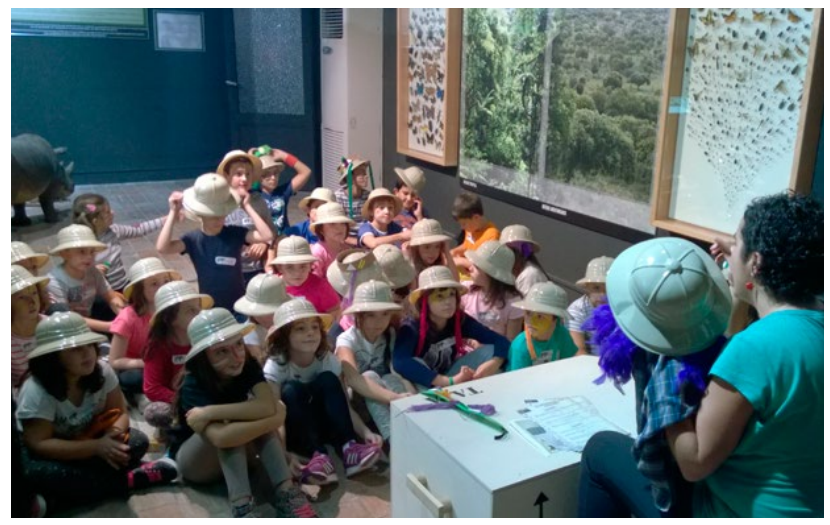

Foto 1: Una noche en el Museo

En el marco de esta celebración, indudablemente el día mas señalado fue el sábado 25 de octubre de 2014, con la culminación de la conmemoración del $\mathrm{X}$ Aniversario con nuevas actividades. La jornada se abrió con la conferencia ¿POR QUÉ CONSERVAR ESPECIES EN PELIGRO?, a cargo del Dr. Miguel Delibes De Castro y Profesor de investigación de la Estación Biológica de Doñana (CSIC), donde desarrolla su experta e intensa labor investigadora sobre la biología y ecología de mamíferos mediterráneos.

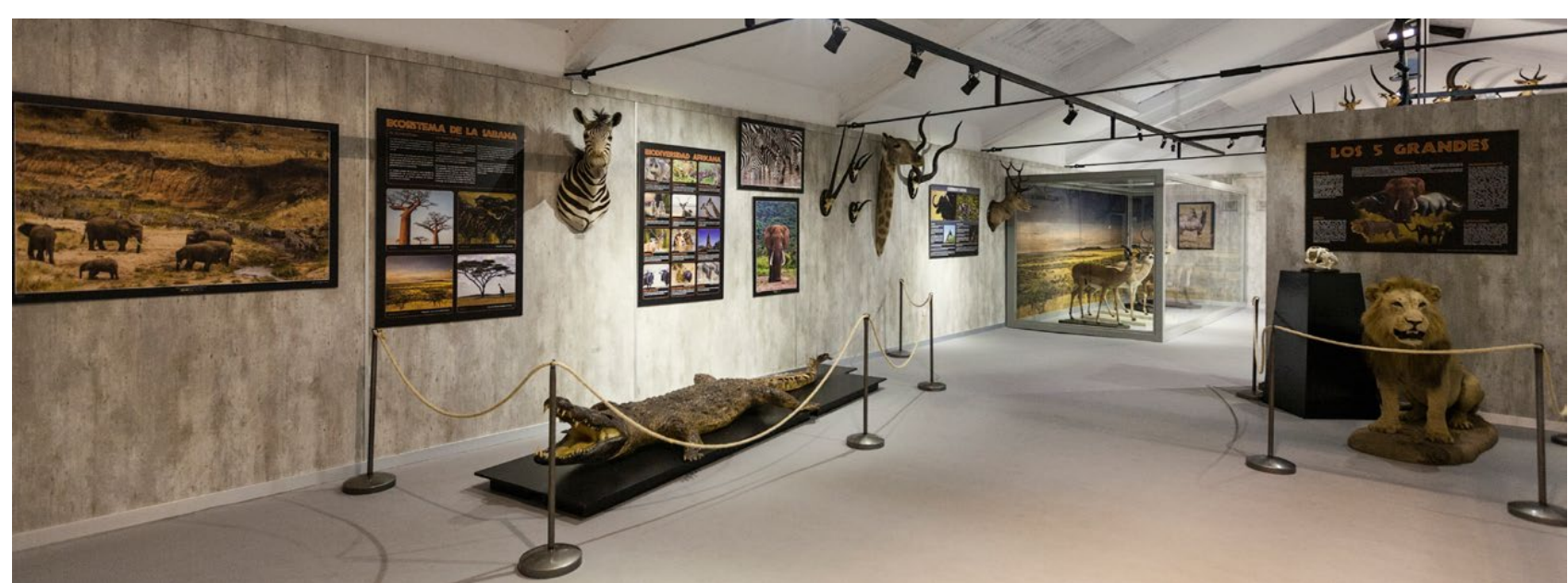

Foto 2: La Sabana africana (Foto: R. Pla)

Esta nueva exposición se ha ofertado a los centros educativos, lo que ha permitido acercar a los escolares a un mundo mágico y con gran biodiversidad como son las grandes sabanas africanas. La respuesta no se hizo esperar por parte de la comunidad educativa y en tan sólo las dos primeras semanas esta nueva exposición ha recibido a más de 760 alumnos.
Seguidamente tuvo lugar el Cuenta Cuentos sobre "LA BIODIVERSDIAD DE LA FONT ROJA" a cargo de Jordi Raúl, que permitió conocer un poco más sobre la biodiversidad del Parque Natural de la Font Roja (Foto 3).

El acto central de la celebración fue presidido por Rafael Serralta Vilaplana, alcalde de Ibi, acompañado por Amparo Navarro Faure, Vicerrectora de Investi- 


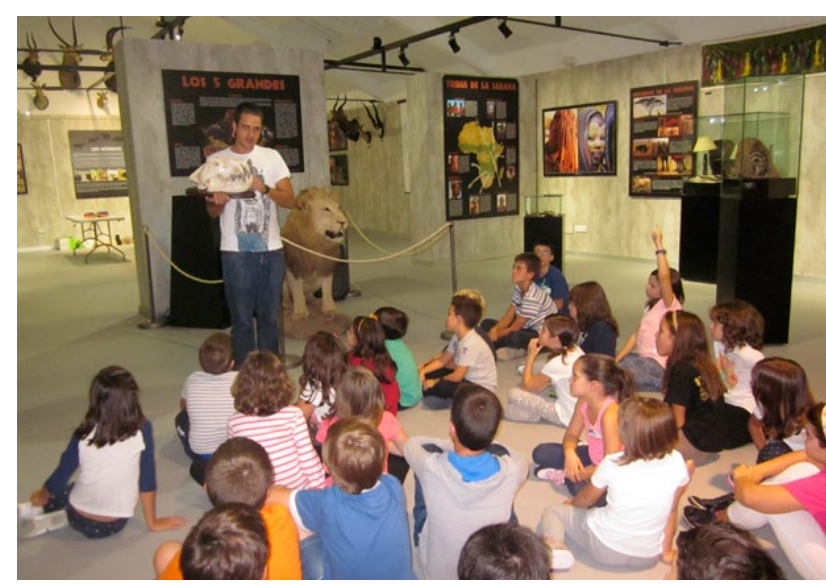

Foto 3: Cuenta cuentos.

gación, Desarrollo e Innovación de la Universidad de Alicante, Ma Ángeles Marcos García, Directora del Instituto Universitario de Investigación CIBIO, Eduardo Galante Patińo, Director del Museo de la Biodiversidad y Francisco Javier Sogorb Guerra, Director Territorial de Infraestructuras, Territorio y Medio Ambiente (Foto 4). Esta intervención de autoridades estuvo seguida por la actuación de la compañía La Luna de África, grupo de danza y percusión que cerró este acto de celebración con un espectáculo de danzas y percusión africana (Foto 5).

Por la tarde el naturalista Luis Miguel Domínguez Mencías, director de documentales y series de televisión, escritor y divulgador del mundo de la naturaleza, cerró la jornada hablando sobre la importancia de la conservación del lobo en Espańa con la conferencia titulada "DEMOS UNA OPORTUNIDAD AL LOBO: el lobo ibérico en la España del siglo XXI".

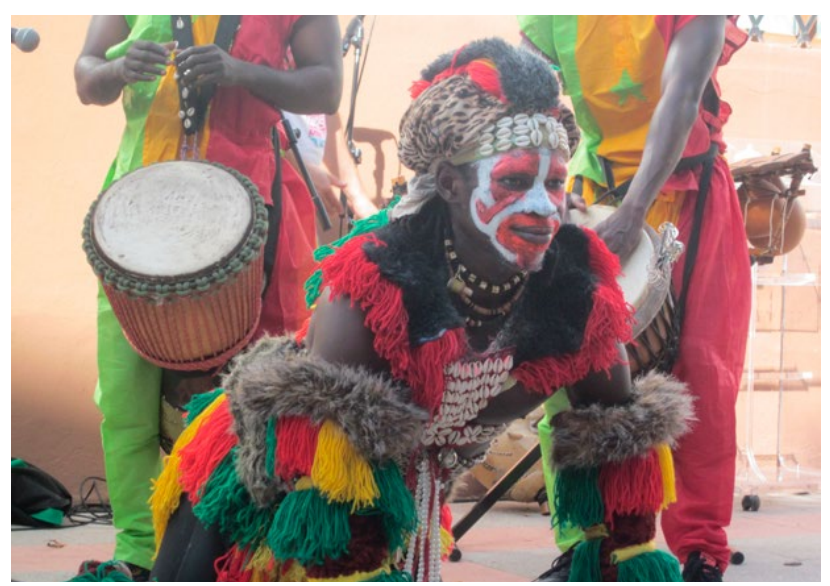

Foto 5: Actuación de la compañía Luna de Africa.

Este feliz cumpleaños terminó con las actuaciones de percusión de la Asociación Trencatoms, con las danzas tradicionales de Ibi, a cargo del Grupo local de Coros y Danzas, con "UN PASSEIG PER IBI I SU BIODIVERSITAT" a cargo de la Asociación Amics de les Muntanyes.

Desde el museo queremos agradecer a las más de 3500 personas que asistieron durante esta primera jornada, a todos los que han colaborado en su organización y hacemos extensiva la invitación a este aniversario a todos aquellos que quieran seguir disfrutando y colaborando en actividades dirigidas a aprender a conservar nuestra biodiversidad a través de las iniciativas que se desarrollan desde el Museo de la Biodiversidad.

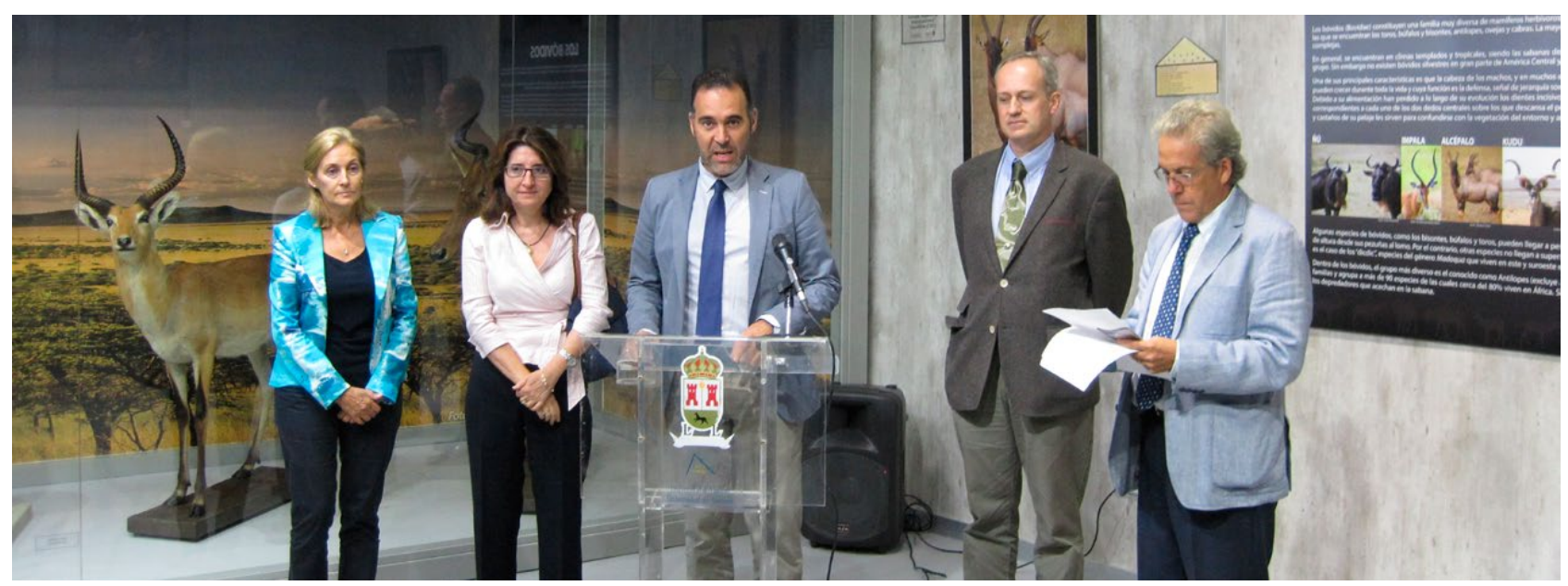

Foto 4: Momento de la inaguración 